\title{
Generation of strut-and-tie models and stress fields for structural concrete
}

\section{Conference Paper}

\section{Author(s):}

Mozaffari, Salma (i); Akbarzadeh, Masoud; Vogel, Thomas

Publication date:

2019-04

Permanent link:

https://doi.org/10.3929/ethz-b-000340330

Rights / license:

In Copyright - Non-Commercial Use Permitted 


\title{
Generation of strut-and-tie models and stress fields for structural concrete
}

\author{
Salma Mozaffari ${ }^{1}$, Masoud Akbarzadeh ${ }^{2}$, and Thomas Vogel ${ }^{3}$ \\ ${ }^{1} \mathrm{PhD}$ Candidate, Institute of Structural Engineering, ETH Zurich, Stefano-Franscini- \\ Platz 5, 8093 Zurich, Switzerland; e-mail: mozaffari@ibk.baug.ethz.ch \\ ${ }^{2}$ Assistant Professor, Polyhedral Structures Laboratory, School of Design, University \\ of Pennsylvania, Pennovation Center, 3401 Grays Ferry Ave., Philadelphia, PA 19146, \\ USA; e-mail:masouda@design.upenn.edu \\ ${ }^{3}$ Professor, Institute of Structural Engineering, ETH Zurich, Stefano-Franscini-Platz 5, \\ 8093 Zurich, Switzerland; e-mail: vogel@ibk.baug.ethz.ch
}

\begin{abstract}
Strut-and-tie models are principally the discretized stress fields, which simplify the dimensioning and detailing of reinforced concrete members. The computational generation of stress fields currently relies on finite element analysis or optimization methods. This research addresses the limitations of optimization algorithms in producing reasonable strut-and-tie configurations. The computational procedure of this paper utilizes layout optimization and graphic statics cooperatively to create strut-andtie models and stress fields for two-dimensional cases. The presented examples demonstrate the capabilities of the mentioned methods to produce some desired results for two-dimensional scenarios and suggest a similar approach to solve the strut-and-tie problem for three-dimensional cases.
\end{abstract}

\section{INTRODUCTION}

The strut-and-tie model (STM) is a truss-like system with a set of compressive struts and tensile ties, which simply represents the load transfer mechanism in a reinforced concrete member, where concrete only contributes to the compression and steel is activated in tension. The model was intuitively developed at the onset of the twentieth century without a theoretical rationale (Ritter 1899 and Mörsch 1906). Its primary goal was to understand the reinforced concrete behavior after cracking. Since the cracked concrete assumed to have no tensile strength, the concept has later found justification in the lower bound theorem of plasticity (Nielsen et al. 1978). Later, useful guidelines were issued leading to popular applications of truss (named as strut-and-tie) models in structural concrete practice (Schlaich et al. 1987). STMs could be considered as discrete representations of stress fields (SFs). The concept of SF was introduced by Drucker (1961) as a direct application of plasticity theory for a simply supported reinforced concrete beam subjected to point and distributed loads. SF and STM are complementary in satisfying equilibrium and yield criteria of the lower bound theorem of plasticity. Therefore, a combination of the methods is typically used in practice to provide safe solutions. The strut-and-tie method has been covered by several concrete 
design guidelines for the dimensioning and detailing of structural concrete; however, the challenge typically is how to construct appropriate configurations. In practice, the model is normally created intuitively and iteratively, similar to the load path method introduced by Schlaich et al. (1987). The use of linear elastic finite element analysis was also proposed by Schlaich et al., where the model is formed by orientating the struts and ties according to the principal stresses.

Over the past two decades, extensive research has been conducted on the development of methodologies to computationally generate mainly two-dimensional strut-and-tie patterns based on the flow of stresses within a structural domain. These studies are based on the various formulations of finite element analysis (Muttoni et al. 2015) or optimization algorithms (Liang et al. 2000, Ali and White 2001). The popular optimization algorithms are based on topology or layout optimization.

Topology optimization addresses the question of material distribution within a structural domain subjected to given load and support conditions in order to achieve a better structural performance, generally a minimized compliance or strain energy (Bendsøe and Kikuchi 1988). Since the resulting pattern is similar to the stress flow inside the structure, it has been used as a backdrop for the orientation of truss elements in the STM (Liang et al. 2000). Layout optimization (LOPT) (Dorn 1964, Fleron 1964), based on the ground structure, assumes a grid of nodes and elements connecting these nodes in a given domain. The result is the optimum size for each element for specified boundary conditions (i.e. load and support). The optimum sizes (i.e. cross sections) below a threshold, normally the ones close to zero, lead to omission of some elements from the initial ground structure. Thus, a final layout of the optimized structure is induced. Since this algorithm produces a truss or a load path inside a structural domain, it has been applied to generate STMs (Ali and White 2001). Figure 1 shows the application of the mentioned optimization methods in the development of STMs.

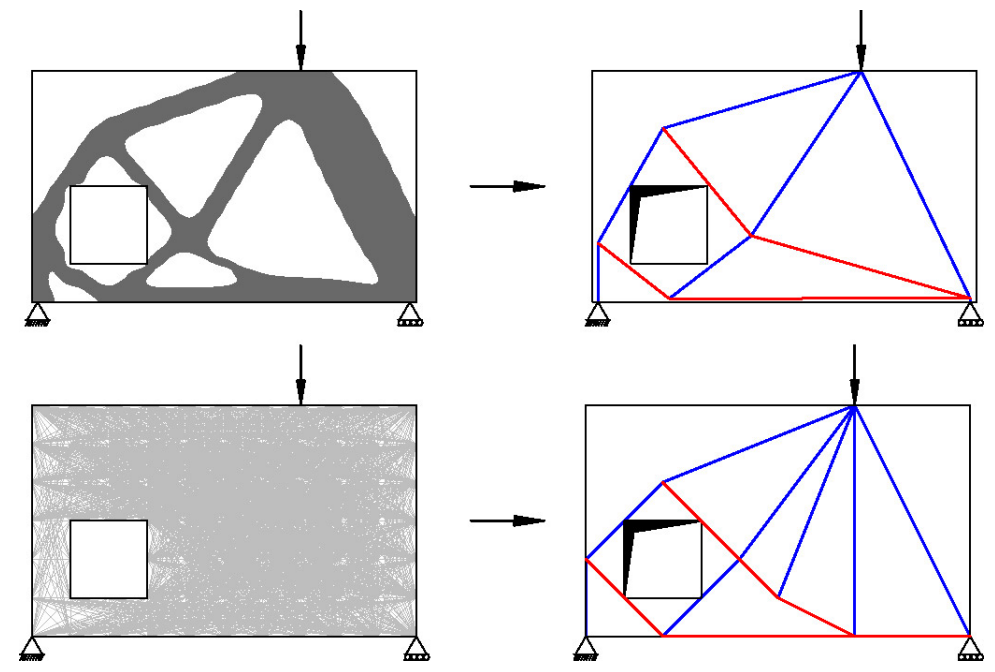

Figure 1: Generation of STM based on: (top) topology optimization (TopOpt for Grasshopper 2018); (bottom) layout optimization. 


\subsection{PROBLEM STATEMENT AND OBJECTIVES}

Although there exist promising results with the computational generation of STMs or SFs using optimization algorithms, narrow focus is given on the practical aspects of the outcomes. The reason could be that these techniques provide single (i.e. the optimized) solutions, where the control over the results cannot always be achieved by manipulation of the constraints. Therefore, these solutions sometimes require further refinements or simplifications to make a useful STM, for example, with proper location, length and orientation of the ties. Moreover, while using topology optimization (e.g. Figure 1 top), an extra effort is required to devise a feasible load path from a material distribution pattern and then calculate the member forces. However, the resulting pattern from LOPT (e.g. Figure 1 bottom) is already a truss in static equilibrium with known member forces.

On the other hand, the geometrical method of graphic statics (GS) provides the flexibility to develop choices of tension-compression truss-like structures, while maintaining the static equilibrium. It functions with the reciprocal form and force diagrams representing the geometry of a truss structure and its force magnitudes, respectively (Culmann 1864 and Maxwell 1864). This technique has recently been computationally developed and used to provide a wide range of typologies for fixed boundary conditions (Lee et al. 2016, Nielsen et al. 2017 and Tabatabaei Ghomi et al. 2018). Furthermore, since the information of the force magnitudes is available geometrically through the force diagram, the discontinuous SFs could be created using Minkowski sum (McRobie 2016).

This study attempts to utilize the potentials of GS to overcome the mentioned inadequacies of the optimization algorithms. One thing to note is, although GS could provide a variety of load path patterns by modifying form or force, an automatic initiation of a pattern accounting for the design domain (i.e. the structure's geometrical boundary) and boundary conditions using GS is not trivial and requires trial and error; Alic and Persson (2018) manually created an initial model to perform a further optimization algorithm on the form and force diagrams to get an initial reinforcement layout. Therefore, in order to be able to use GS, an initial model is required, which we suggest to obtain from an LOPT algorithm (Ali and White 2001).

The ultimate goal of this research is to provide educators and practitioners with a range of useful STM solutions, where each is evaluated based on criteria such as reinforcement volume, fabrication, durability, etc.

This paper presents a methodology to combine the formulations of LOPT and algebraic graphic statics (AGS) (Van Mele et al. 2014) to provide an initial truss model (form diagram) and its corresponding force diagram. The geometric Minkowski sum is used to combine the form and force diagrams to create discontinuous stress fields (McRobie 2016). The further investigation on the potentials of GS for modification of the outputs or providing more solutions for a defined problem and application of the same strategy to three-dimensional problems are work in progress. 


\section{METHODOLOGY}

As mentioned, since the initiation of an STM accounting for the design domain and boundary conditions using GS requires trial and error, an initial model could be provided using LOPT. In this section, we first provide the basic formulation of LOPT and AGS separately, then introduce a combined version to produce initial form and force diagrams for a given domain and boundary condition. The Python programming language (2016) is used for the computational implementation. For the graph manipulations, the COMPAS framework has been utilized (2017).

\subsection{LAYOUT OPTIMIZATION}

A basic LOPT formulation is a linear programming defined as (variables in bold):

$$
\begin{aligned}
& \min V=l^{T} \boldsymbol{a} \\
& \text { subject to: } \\
& \quad B \boldsymbol{f}=F \\
& -\sigma_{C} \boldsymbol{a} \leq \boldsymbol{f} \leq \sigma_{T} \boldsymbol{a} \\
& \boldsymbol{a} \geq 0 \\
& \boldsymbol{f}_{\text {diag }} \geq 0
\end{aligned}
$$

where $V$ is the total volume of the structure, $l$ and $a$ are the vectors of length and crosssectional area of the members, $(1 \mathrm{~b})$ is the equilibrium condition constraint with $B$ as the cosine-connectivity matrix, $f$ the member forces vector and $F$ the external loads vector, (1c) is the stress constraint with $\sigma_{C}$ and $\sigma_{T}$ as factored yield stresses in compression and tension, (1d) limits the areas to non-negative values and (1e) is an additional constraint on the diagonal member forces to only carry compression, which aids in producing simpler truss patterns with no crossing diagonals.

The objective in (1a) is to minimize the total volume of the structure subjected to (1b)-(1e) constraints. The outputs of the optimization are $f$ and $a$ vectors and the minimized objective value (i.e. the total volume $V$ ). The members with zero or very small cross sectional areas will be removed leading to a truss pattern as a subset of the original ground truss (see Figure 2). The first order ground truss is chosen here.

It should be noted that the objective function in (1a) is a reformulation of the objective function in Beghini et al. (2014) and Alic and Persson (2018), where instead of a ground truss, an initial determinate truss is manually devised from topology optimization and linear elastic finite element analysis, respectively. 

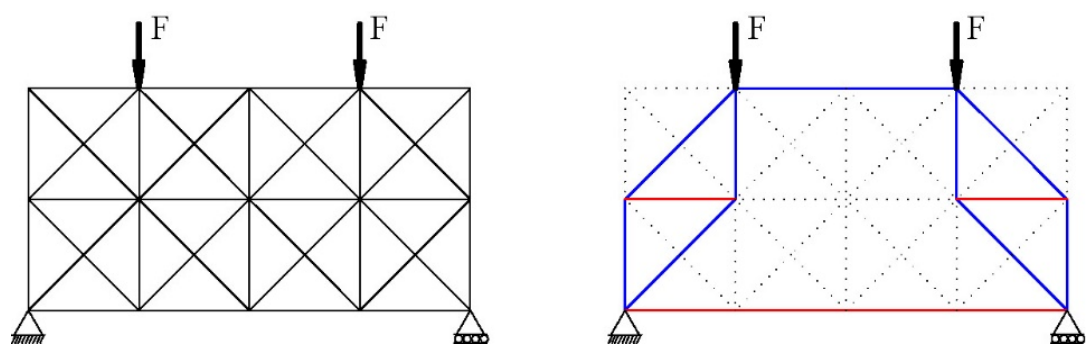

Figure 2: Layout optimization: (left) input ground truss; (right) output optimized truss (blue: compression, tension: red, dashed: zero-area).

\subsection{ALGEBRAIC GRAPHIC STATICS}

The AGS formulation makes it possible to develop the reciprocal relationship between the form and force diagrams computationally, based on the structural matrix analysis and the graph theory. The detailed steps are explained in Van Mele and Block (2014). Here, we repeat two of the main equations, leading to calculation of the forces using the equilibrium condition in (2a) and node coordinates of the force diagram in (2b) for a given form diagram (i.e. the truss geometry):

$$
\begin{aligned}
& A q=0 \\
& X^{*}=L^{*-1} C^{*} Q U \\
& L^{*}=C^{*} C^{* T}
\end{aligned}
$$

where $A$ is the equilibrium matrix, $q$ is the vector of member force densities (i.e. force divided by length), $X^{*}$ and $C^{*}$ are the coordinate and connectivity matrix of the force diagram, $Q$ is the diagonal matrix of vector $q$ and $U$ is the coordinate difference matrix of the form diagram.

By solving (2a) with taking the known elements of $q, q_{i d}$, and its corresponding partitions of $A$ to the right side, the unknown force density vector, $q_{d}$, is calculated. By constructing the complete $q$ vector and substituting $Q$ in $(2 \mathrm{~b}), X^{*}$, the node coordinate matrix of the force diagram, is obtained ( $C^{*}$ and $U$ are known, once the truss geometry is given). Having $X^{*}$ and $C^{*}$, one can plot the force diagram as it is shown in Figure 3. The Bow's notation (1873) shows the reciprocal relationship between the truss elements in the form diagram and their force magnitudes in the force diagram. 

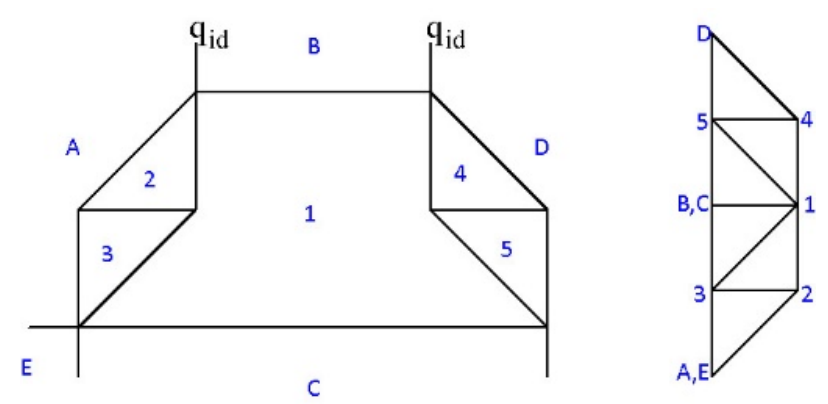

Figure 3: Algebraic graphic statics: (left) input graph (form diagram); (right) output dual graph (force diagram) (Bow's notation in blue (1873)).

\subsection{COMBINED ALGORITHM}

As it is observed, the equilibrium conditions in LOPT and AGS, (1b) and (2a), allow the calculation of the forces (or force densities). Also, in order to generate the force diagram, the optimized truss (Figure 2 right) is used as input for AGS (Figure 3 left). However, if the output is an indeterminate structure, extra effort (i.e. finding the independent edges and assigning them the correct densities) is required to produce similar force densities as optimization. The combined formulation avoids the double consideration of the equilibrium condition by calculating the force densities in the optimization process. The force densities are directly used in the second equation of AGS, where there is no need to find the independent edges in the case of an indeterminate pattern.

In order to replace the force vector, $f$, in (1b) with the force density vector, $q_{d}$, matrix $A_{n}$ is created, where the rows related to the fixed directions (i.e. support directions) and the columns related to external load/support edges have been removed from matrix $A$ in (2a). The combined algorithm follows (variables in bold):

$$
\begin{aligned}
& \min V=l^{T} \boldsymbol{a} \\
& \text { subject to: } \\
& \quad A_{n} \boldsymbol{q}_{\boldsymbol{d}}=F \\
& \quad-\sigma_{C} \boldsymbol{a} \leq \boldsymbol{q}_{\boldsymbol{d}} \odot l \leq \sigma_{T} \boldsymbol{a} \\
& \quad \boldsymbol{a} \geq 0 \\
& \quad \boldsymbol{q}_{\boldsymbol{d}, \boldsymbol{d i a g}} \geq 0 \\
& X^{*}=L^{*-1} C^{*} Q U
\end{aligned}
$$

where the term $q_{d} \odot l$ is the element-wise product of the force density and length vectors. The optimization is performed with the constraints (3b)-(3e). Now, that the truss geometry and force densities are known, the coordinates of the force diagram can be calculated with (3f). 
In the next step, a visualization of discontinuous SFs (including hydrostatic nodes) is possible by scaling the force diagram proportional to the concrete's factored compression strength $\left(f_{c}\right)$ and adding it to the form diagram using the Minkowski sum. This is an advantage of having both diagrams through GS. Figure 4 shows the schematic input/outputs for the combined algorithm including the resulting SFs.

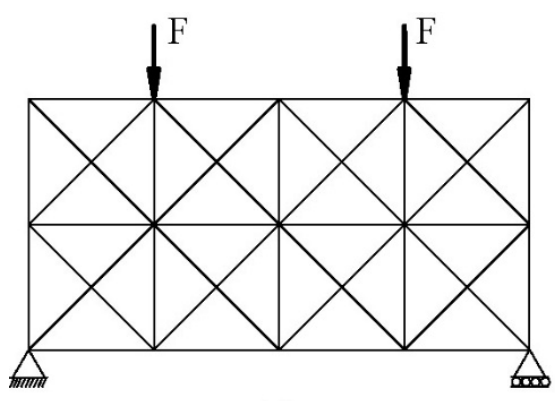

(1)

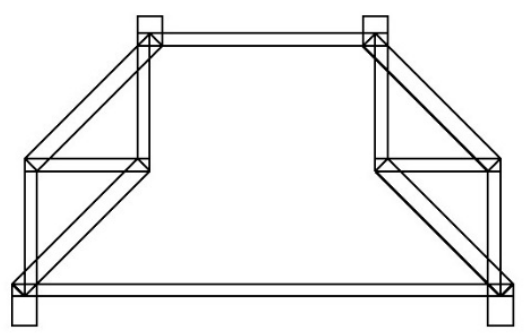

(3)

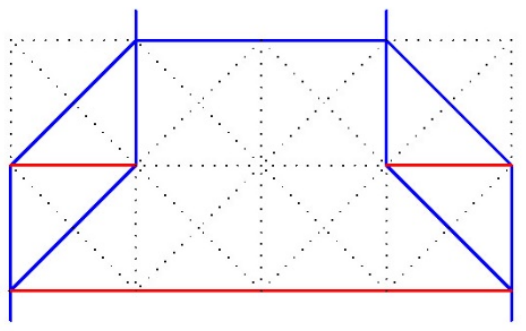

(2)
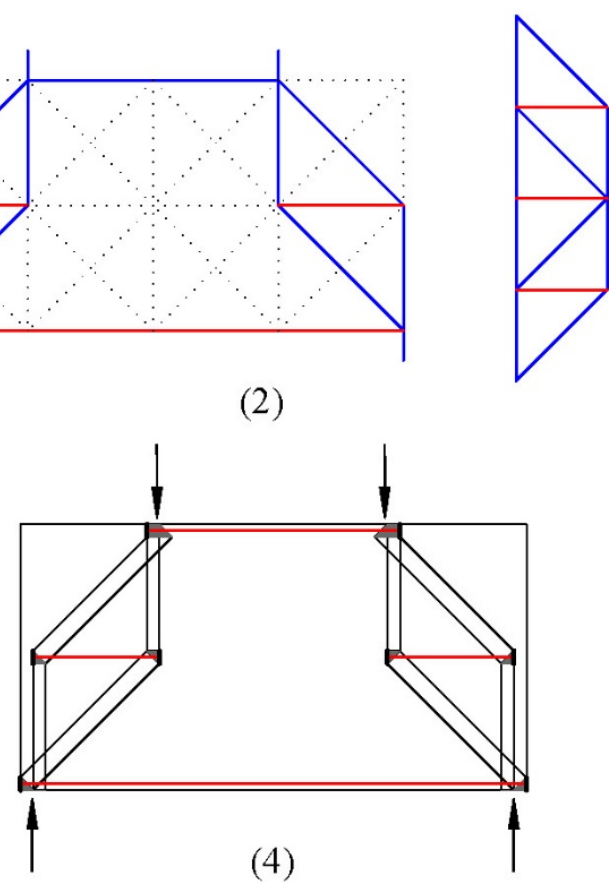

Figure 4: (1) Input truss/boundary condition; (2) output form and force diagrams; (3) Minkowski sum of the diagrams; (4) conventional discontinuous stress fields.

\section{EXAMPLES}

Figures 5 and 6 show the application of the method for several boundary conditions. When the geometry is regular or without opening, the solutions look simpler. Some solutions in Figures 5 or 6 may not directly be usable as an STM. For example, models 3 and 4 in Figure 6 need simplifications. Also, the first models in Figures 5 and 6 with direct load transfer to the supports and long tension paths are undesirable. An admissible STM is simple, has a reasonable load transfer pattern with vertical ties for shear transfer (i.e. it can make use of the minimum reinforcement) and shorter reinforcement lengths (e.g. models 2 and 4 in Figure 5) (Kaufmann 2018).

The examples provided here confirm that in many cases, relying only on optimization to generate a STM for the purpose of reinforced concrete design is not sufficient. The control over the optimization solution is not always achievable by 
manipulation of the constraints. Therefore, sometimes a next step is required to refine, rationalize or simplify the output patterns.
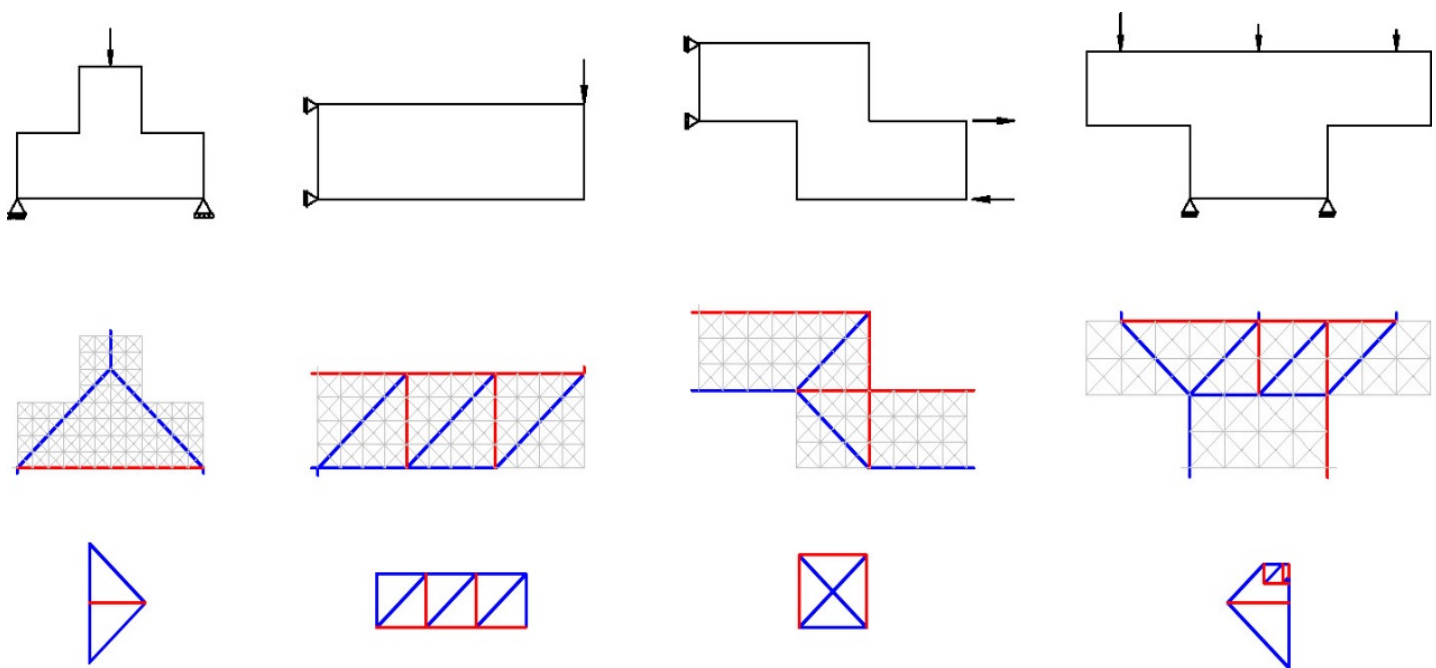

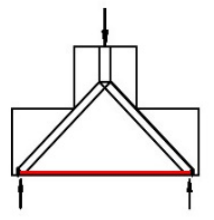

(1)

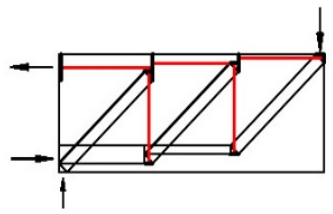

(2)

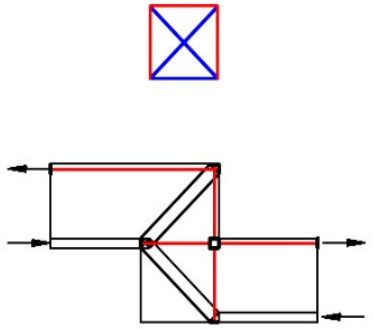

(3)

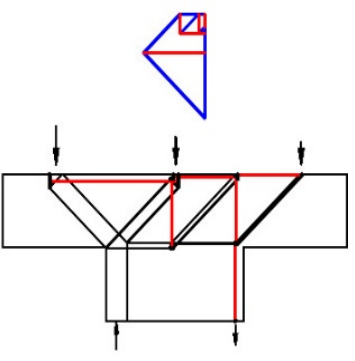

(4)

Figure 5: Application to boundaries with regular geometry: (top to bottom) boundary condition; optimized truss (form diagram); force diagram; discontinuous stress fields.

\section{DISCUSSION AND OUTLOOK}

An optimization algorithm is combined with the algebraic formulation of graphic static to help generate initial STMs and discontinuous SFs. The presented examples indicate that the optimization solutions are not always satisfying. The irregularities in the domain, such as openings or non-rectangular shapes including the imposed initial mesh (i.e. the ground truss) could lead to complex models. On the other hand, simple models with direct load transfer patterns, long ties and no shear transfer mechanisms are less desirable for our purpose. Thus, graphic statics will be utilized to set up a postprocessing procedure on the initial outputs and possibly provide few useful STM options for a given problem. Most importantly, this research aims to contribute further to the generation of appropriate three-dimensional STMs. The created models could be used in education and practice or be integrated into the newly developed software by Muttoni et al. (2015) and Mata-Falcon et al. (2018). These tools develop realistic continuous stress fields, but still require one (or more) valid STM(s) as a starting point to input the reinforcement pattern. 

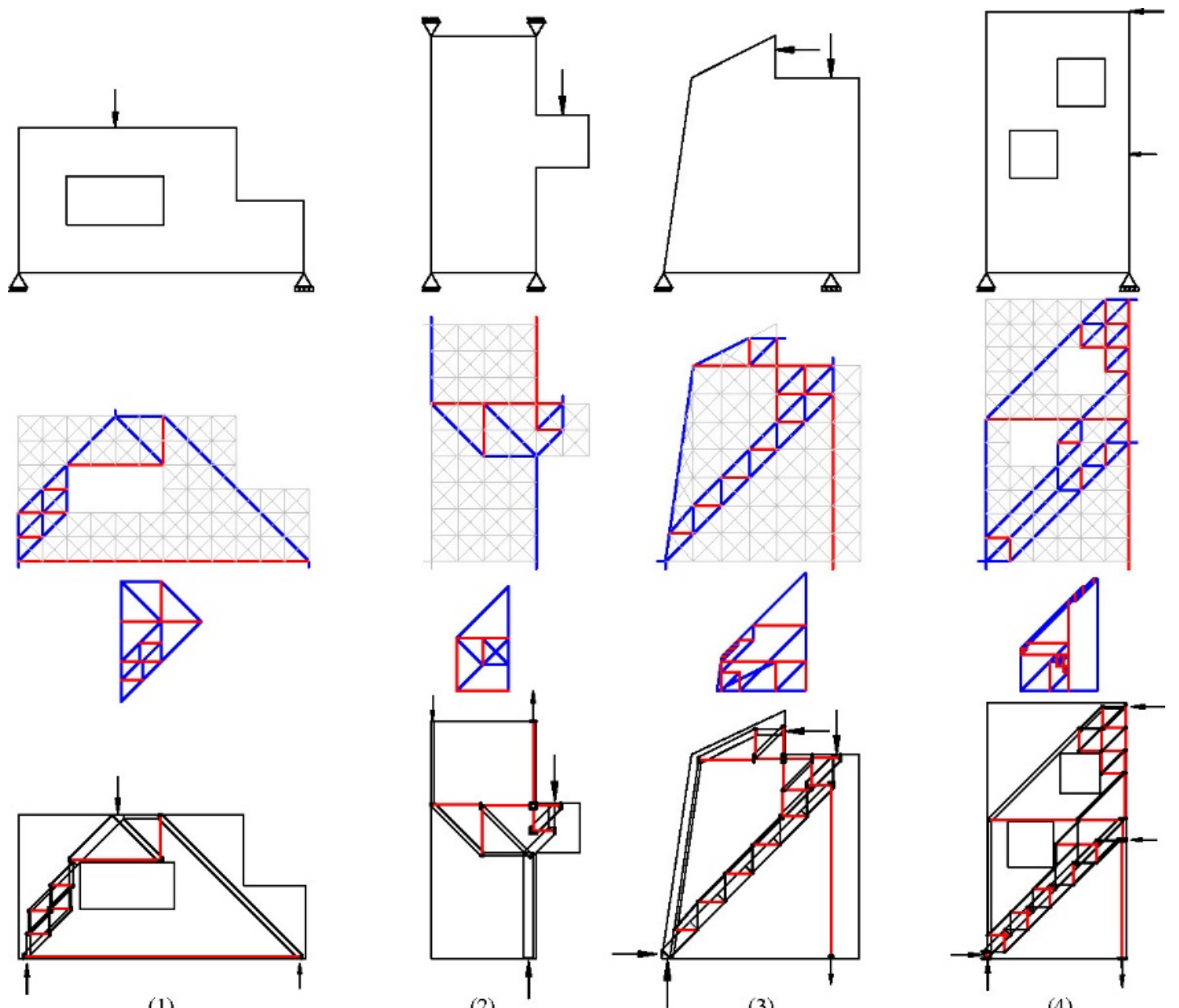

(1)

(2)

(3)

(4)

Figure 6: Application to boundaries with irregular geometry: (top to bottom) boundary condition; optimized truss (form diagram); force diagram; discontinuous stress fields.

\section{REFERENCES}

Ali, M. and White, R. (2001). "Automatic generation of truss model for optimal design of reinforced concrete structures." Structural Journal, 98(4), 431-442.

Alic, V. and Persson, K. (2018). "Generating initial reinforcement layouts using graphic statics." Proc., IASS Annual Symposia.

Beghini, L., Carrion, J., Beghini, A., Mazurek, A., and Baker, W. (2014). "Structural Optimization using Graphic Statics." Structural and Multidisciplinary Optimization, 49(3), 351-366.

Bendsøe, M. P. and Kikuchi, N. (1988). "Generating optimal topologies in structural design using a homogenization method." Computer Methods in Applied Mechanics and Engineering, 71(2), 197-224. 
Bow, R. H. (1873). Economics of construction in relation to framed structures. Spon, London.

Culmann, K. (1864). Die Graphische Statik. Verlag Meyer und Zeller, Zürich.

Dorn, W. (1964). “Automatic design of optimal structures." Journal de Mecanique, 3, $25-52$.

Drucker, D. (1961). "On structural concrete and the theorems of limit analysis." IABSE Publications, 21, 49-59.

Fleron, P. (1964). "Minimum weight of trusses." Bygningsstatiske Meddelelser, 35(3), 81.

Kaufmann, W. (2018). Stahlbeton III.

$<$ http://www.kaufmann.ibk.ethz.ch/lehre/masterstudium/stahlbeton-iii.html $>$ (Oct. 15, 2018).

Lee, J., Van Mele, T., and Block, P. (2016). "Form-finding explorations through geometric transformations and modifications of force polyhedrons." Proc., IASS Annual Symposia, 15, 1-10.

Liang, Q., Xie, Y., and Steven, G. (2000). "Topology optimization of strut-and-tie models in reinforced concrete structures using an evolutionary procedure." $A C I$ Structural Journal, 97(2), 322-332.

Mata-Falcón, J., Tran, D. T., Kaufmann, W., and Navrátil, J. (2018). "Computer-aided stress field analysis of discontinuity concrete regions." Proc., Computational Modelling of Concrete and Concrete Structures (EURO-C 2018), 641-650.

Maxwell, J. (1864). "On reciprocal figures and diagrams of forces." Philosophical Magazine and Journal Series, 4(27), 250-261.

McRobie, A. (2016). "Maxwell and Rankine reciprocal diagrams via Minkowski sums for two-dimensional and three-dimensional trusses under load." International Journal of Space Structures, 31(2-4), 203-216.

Mörsch, E. (1906). Der Eisenbetonbau, seine Theorie und Anwendung. K. Wittwer, Stuttgart.

Muttoni, A., Fernández Ruiz, M., and Niketic, F. (2015). "Design versus assessment of concrete structures using stress fields and strut-and-tie models." ACI Structural Journal, 112.

Nielsen, M., Braestrup, M., Jensen, B., and Bach, F. (1978). "Concrete plasticity, beam shear-shear in joints-punching shear." Special Publication, Danish Society for Structural Science and Engineering, 1-129.

Neilsen, T., Akbarzadeh, M., and Goltermann, P. (2017). "Addressing buckling of compression members using subdivision of force diagrams." Proc., IASS Annual Symposia.

Python software foundation. Python Language Reference, version 2.7.13, (2016). $<$ https://www.python.org/> (Oct. 15, 2018).

Ritter, W. (1899). "Die Bauweise Hennebique." Schweizerische Bauzeitung, 33(7), $41-61$.

Schlaich, J., Schäfer, K., and Jennewein, M. (1987). "Toward a consistent design of structural concrete.” Precast Concrete Institute (PCI) Journal, 32(3), 74-150. 
Tabatabaie ghomi, A., Bolhassani, M., Nejur, A., and Akbarzadeh, M. (2018). "Effect of subdivision of force diagrams on the local buckling, load-path and material use of founded forms." Proc., IASS Annual Symposia.

TopOpt plugin for Rhino and Grasshopper (2018). $<$ http://www.topopt.mek.dtu.dk/Apps-and-software/TopOpt-plugin-for-Rhinoand-Grasshopper $>$ (Nov. 12, 2018).

Van Mele, T. and Block, P. (2014). "Algebraic graph statics." Computer-Aided Design, 53, 104-116.

Van Mele, T., Liew, A., Mendéz, T., and Rippmann, M. (2017). COMPAS: A framework for computational research in architecture and structures.

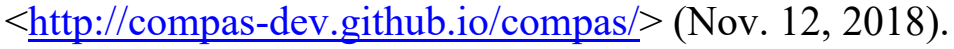

